\title{
Identification of a FUS splicing mutation in a large family with amyotrophic lateral sclerosis
}

\author{
Véronique V Belzil ${ }^{1}$, Judith St-Onge ${ }^{1}$, Hussein Daoud ${ }^{1}$, Anne Desjarlais ${ }^{1}$, Jean-Pierre Bouchard ${ }^{2}$, \\ Nicolas Dupré2, William Camu ${ }^{3}$, Patrick A Dion ${ }^{1,4}$ and Guy A Rouleau ${ }^{1,5,6}$
}

Amyotrophic lateral sclerosis (ALS) is a severe neurodegenerative disease characterized by the degeneration of upper and lower motor neurons. Genetic studies have led, thus far, to the identification of 12 loci and 9 genes for familial ALS (FALS). Although the distribution and impact of superoxide dismutase 1 mutations has been extensively examined for over a decade, the recently identified FALS-associated FUS gene has been less studied. Therefore, we set out to screen our collection of FALS cases for FUS mutations. All 15 exons of FUS were amplified and sequenced in 154 unrelated FALS cases and 475 ethnically matched healthy individuals. One substitution located in the acceptor splice site of intron 14 was identified in all affected members of a large family, causing the skipping of the last 13 amino acids of the protein and the translation of 7 novel amino acids, resulting from the new translation of a part of the $3^{\prime}$ untranslated region. Our study identified a new splicing mutation in the highly conserved C-terminal of the FUS protein. Thus far most FUS mutations are missenses, and our findings, combined with those of others, confirm the importance of the C-terminal portion of the protein, adding additional support for FUS mutations having a critical role in ALS.

Journal of Human Genetics (2011) 56, 247-249; doi:10.1038/jhg.2010.162; published online 16 December 2010

Keywords: amyotrophic lateral sclerosis; FUS/motor neuron disease; neurodegeneration; RNA-binding protein; splicing mutation

Amyotrophic lateral sclerosis (ALS) is a neurodegenerative disease affecting the upper and lower motor neurons. Specifically, neurons of the motor cortex, brain stem and spinal cord are progressively involved, causing gradual spasticity and muscle weakness starting in the limbs in $75 \%$ of cases and in the bulbar region in $25 \%$ of cases. In all, $90 \%$ of patients are believed to be sporadic (SALS), without any family history of the disease, while the other $10 \%$ of cases are familial (FALS), primarily segregating in an autosomal dominant manner. ${ }^{1}$ SALS and FALS patients are clinically indistinguishable, except for the mean age of onset, which is 56 years for SALS, compared with an average of 46 years for FALS. $^{2}$ The overall prevalence is $4-6 / 100000$ and incidence is $1-2 / 100000,{ }^{3}$ which make ALS the most common of motor neuron diseases.

Mutations in the copper superoxide dismutase 1 gene were first shown to be ALS causative over 15 years ago and they account for $15-20 \%$ of all FALS cases, representing a proportion of $1-2 \%$ of all ALS cases. ${ }^{4}$ In the last 2 years, the identification of ALS causative mutations in the TAR-DNA-binding protein (TARDBP encoding TDP-43) gene in both SALS and FALS cases $^{5}$ and in FUS/TLS encoding FUS, ${ }^{6,7}$ thus far mostly but not exclusively in FALS, opened a new era for the investigation of mechanisms underlying the disease. Whereas superoxide dismutase 1 mutations have been reported throughout the full length of the protein, TARDBP and FUS mutations are mostly clustered to specific regions. For TARDBP, most of the mutations identified are in the glycine-rich region encoded by exon 6 , and for FUS/TLS, which also contains a glycine-rich region, mutations are mostly in the extreme C-terminal part of the protein. ${ }^{8}$ The aim of this study was to evaluate the proportion of FUS mutations in a portion of our FALS cohort and to see if any mutations identified would be clustered to the same region.

The 15 coding exons of FUS were amplified and sequenced in 154 unrelated FALS cases, and only one variant was identified. It is an unreported substitution of an adenine to a cytosine in the acceptor splice site of intron 14 (c.1542-2A >C). The variant was present in six samples of a large ALS family (Figure 1) recruited in France: II:5, III:8, IV:1, IV:3, IV:4, and IV:6. Two of those individuals had an ALS phenotype (II:5, III:8), while the four unaffected mutation carriers are in the fourth generation and are younger than the average age of onset of the first ALS symptoms. By carrying the mutation, they confirm that their three affected fathers likely had the substitution. In addition, three mothers married to affected fathers (II:4, III:1 and III:7) were tested and were negative for the mutation. We prepared cDNA using total RNA from the immortalized lymphoblast cell lines of the two affected individuals with the adenine-to-cytosine

${ }^{1}$ Center of Excellence in Neuromics of Université de Montréal, CHUM Research Center, Montreal, Quebec, Canada; ${ }^{2}$ Université Laval, Faculty of Medicine, CHA, Enfant-Jésus Hospital, Quebec, Canada; ${ }^{3}$ ALS Center, Department of Neurology, CHU Gui de Chauliac, Montpellier, France; ${ }^{4}$ Department of Pathology and Cellular Biology, Faculty of Medicine, Université de Montréal, Montreal, Quebec, Canada; ${ }^{5}$ Department of Medicine, Faculty of Medicine, Université de Montreal, Montreal, Quebec, Canada and ${ }^{6}$ Research Center, CHU Sainte-Justine, and Department of Pediatrics and Biochemistry, Université de Montreal, Montreal, Quebec, Canada

Correspondence: Dr GA Rouleau, CHUM Research Centre, Notre-Dame Hospital, 1560 Sherbrooke East, Y-3633, Montreal, Quebec, Canada H2L 4M1.

E-mail: guy.rouleau@umontreal.ca

Received 30 October 2010; revised 18 November 2010; accepted 26 November 2010; published online 16 December 2010 


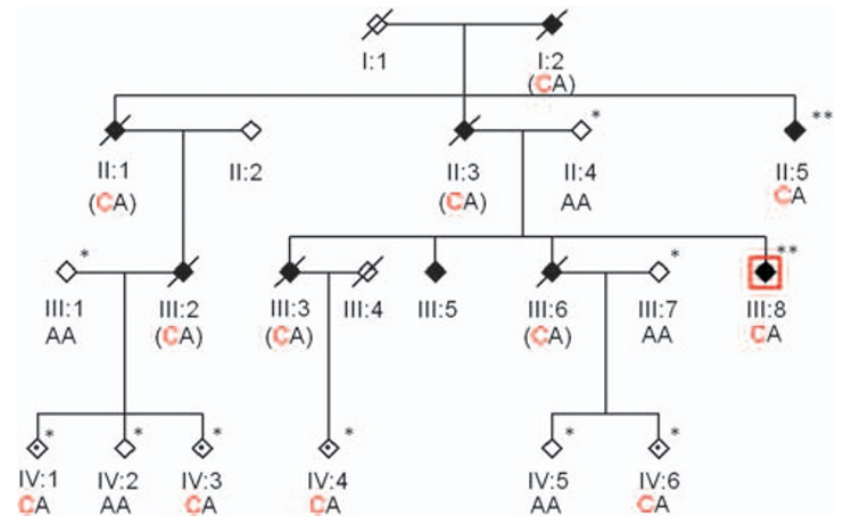

Figure 1 Pedigree of the family with the c.1542-2A $>C$ variant. Black symbols represent affected individuals, while open symbols represent unaffected family members. Symbols with dots represent unaffected individuals carrying the substitution. Slashed symbols indicate deceased individuals. A red square around the individual III:8 symbol indicates the sample sequenced in the primary screening, and asterisks indicate family members for whom exon 15 of the FUS gene was sequenced in the secondary screening. Symbols with two asterisks represent individuals for whom cDNA was amplified. Genotypes at position c.1542-2 are indicated in black for the wild-type allele and in red for the mutant allele. Genotypes in parentheses are inferred. substitution. These cDNAs were PCR amplified, and two products of 425 and $167 \mathrm{bp}$ were observed on agarose gel (Figure 2a), demonstrating that the mutant allele was not degraded by nonsense-mediated mRNA decay. The heterozygous sequence trace was analyzed, as well as the separate sequence of the two different alleles after a gel extraction of the two products (Figure $2 \mathrm{~b}$ ). Sequencing showed that patients with the c.1542-2A $>C$ change expressed a mutated allele missing the $40 \mathrm{bp}$ coding sequence of exon 15 as well as the first $203 \mathrm{bp}$ of the $3^{\prime}$ untranslated region. More precisely, the sequence of the mutant mRNA brings together the last $2 \mathrm{bp}$ of amino acid 514 located in exon 14 and the nucleotide at the c. ${ }^{\star} 204$ position in the $3^{\prime}$ untranslated region, as an AG alternative acceptor site located at position c. ${ }^{\star 202}{ }^{\star} 203$ is encountered (Figure $2 c$ ); translation subsequently ends with a stop codon at position c. ${ }^{\star} 228$. Consequently, the mutant protein undergoes a silent change at amino acid 514 (p.R514R), followed by seven new amino acids (SMSRSGR) at the end of the protein. The adenine of the wild-type acceptor site of intron 14 located at base pair 31110219 on chromosome 16 is highly conserved across species and is not listed as a known SNP. The Alternative Splice Site Predictor bioinformatics program attributed a score of 15.533 to the wild-type AG acceptor splice site, and a score of 4.249 for the acceptor splice site with the cytosine substitution. The alternative AG acceptor splice site located in the $3^{\prime}$ untranslated region at position c. ${ }^{\star} 202 \_$¿ 203 had a score of 4.620 and is suggested to
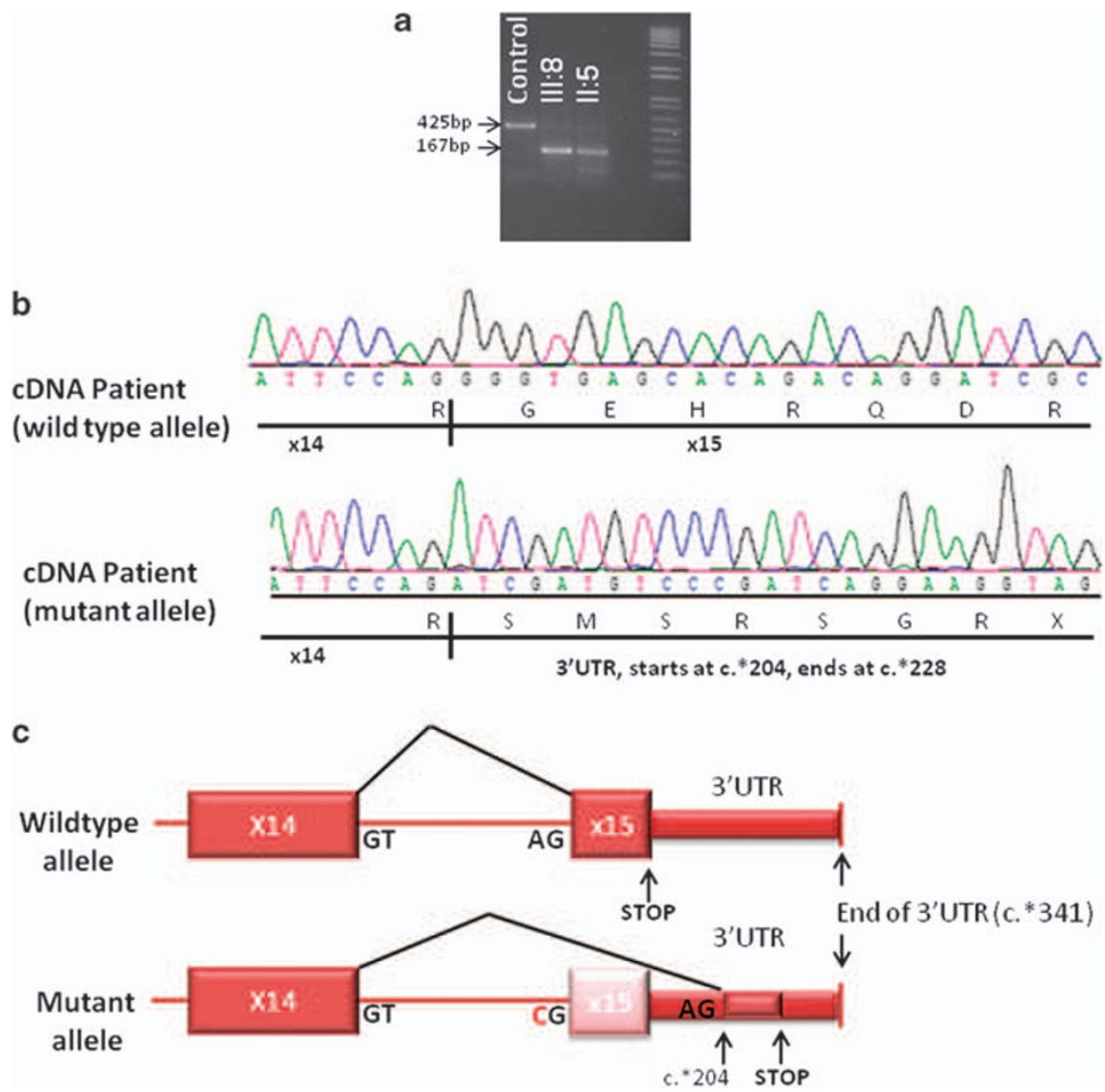

Figure 2 Splicing mutation in FUS. (a) Agarose gel electrophoresis of FUS cDNA amplified in one unrelated control, and two affected family members (II:5, III:8), showing the wild-type allele ( $425 \mathrm{bp}$ ) and the mutant allele (167 bp) for the fragment amplified using foward primer $5^{\prime}$-GAGGGGG ACCAGGTGGCTCTCAC in exon 14 and reverse primer $5^{\prime}$-TCATTTGGCCTTCTCCCCGAACAC in the $3^{\prime}$ untranslated region (UTR). (b) cDNA sequence chromatogram of the wild-type and mutant alleles in one patient, confirming the skipping of exon 15 and the first 203 bp of the $3^{\prime}$ UTR. (c) Schematic (not to scale) representation of exons 14, 15 and the 3'UTR of the FUS gene for the wild-type and mutant alleles. In the mutant allele, a part of the 3'UTR is translated, which adds seven new amino acids to the protein (SMSRSGR). 
be responsible for this new isoform. As this FUS mutation segregates in all affected individuals of the family as well as in the children of deceased affected members, and was not present in 475 control participants, we believe that it is likely responsible for the ALS phenotype observed in this family.

The substitution is located in the RGG domain enriched in arginine-glycine-glycine motifs, where most other FUS mutations were reported. Importantly, truncated proteins were previously observed with the two other major ALS causative genes. ${ }^{9-11}$ But, more interestingly, this is the second splicing mutation reported in FUS, as another one was recently shown to lead to the skipping of exon 14 after the substitution of the adenine located in the acceptor splice site of intron $13 .{ }^{12}$ Unfortunately, no clinical information could be retrieved for the family presented in our study, so we were unable to compare the disease evolution caused by our splicing mutation in intron 14 with the splicing mutation in intron 13 reported by DejesusHernandez et al. ${ }^{12}$ The splicing mutation identified here removes the last 13 amino acids that partly encode the C-terminal portion of FUS, in which a number of mutations have been associated with ALS. Our finding reinforces the notion that this portion has a key role in the pathology of ALS, most likely by way of affecting the RNAbinding domain it encodes for. In the future, such splicing defect causative mutations may become treatable through drugs or gene therapies aimed at correcting splicing, as explored for a number of disorders. ${ }^{13-15}$

\section{ACKNOWLEDGEMENTS}

VVB, HD and GAR are supported by the Canadian Institutes of Health Research. We would like to thank the patients involved in this study; Mélanie Benard, Isabelle Thibault and Pierre Provencher for sample collection and organization; Anne Noreau, Cynthia Bourassa, Sophie Massart and Bertrand Boutié for technical support; and to acknowledge support from the Association pour la Recherche sur la Sclérose Latérale Amyotrophique (ARS), the Association Française contre les Myopathies (AFM) and the French Group on MND.
1 Shaw, C. E., Enayat, Z. E., Powell, J. F., Anderson, V. E., Radunovic, A., Al-Sarraj, S. et al. Familial amyotrophic lateral sclerosis. Molecular pathology of a patient with a SOD1 mutation. Neurology 49, 1612-1616 (1997).

2 Camu, W., Khoris, J., Moulard, B., Salachas, F., Briolotti, V., Rouleau, G. A. et al. Genetics of familial ALS and consequences for diagnosis. French ALS Research Group. J. Neurol. Sci. 165(Suppl 1), S21-S26 (1999).

3 Kurtzke, J. F. Epidemiology of amyotrophic lateral sclerosis. Adv. Neurol. 36, 281-302 (1982).

4 Rosen, D. R., Siddique, T., Patterson, D., Figlewicz, D. A., Sapp, P., Hentati, A. et al. Mutations in $\mathrm{Cu} / \mathrm{Zn}$ superoxide dismutase gene are associated with familial amyotrophic lateral sclerosis. Nature 362, 59-62 (1993).

5 Sreedharan, J., Blair, I. P., Tripathi, V. B., Hu, X., Vance, C., Rogelj, B. et al. TDP-43 mutations in familial and sporadic amyotrophic lateral sclerosis. Science 319, 1668-1672 (2008).

6 Kwiatkowski, T. J. Jr, Bosco, D. A., Leclerc, A. L., Tamrazian, E., Vanderburg, C. R. Russ, C. et al. Mutations in the FUS/TLS gene on chromosome 16 cause familial amyotrophic lateral sclerosis. Science 323, 1205-1208 (2009).

7 Vance, C., Rogelj, B., Hortobagyi, T., De Vos, K. J., Nishimura, A. L., Sreedharan, J. et al. Mutations in FUS, an RNA processing protein, cause familial amyotrophic lateral sclerosis type 6. Science 323, 1208-1211 (2009).

8 Lagier-Tourenne, C. \& Cleveland, D. W. Rethinking ALS: the FUS about TDP-43. Cell 136, 1001-1004 (2009).

9 Daoud, H., Valdmanis, P. N., Kabashi, E., Dion, P., Dupre, N., Camu, W. et al. Contribution of TARDBP mutations to sporadic amyotrophic lateral sclerosis. J. Med. Genet. 46, 112-114 (2009).

10 Valdmanis, P. N., Belzil, V. V., Lee, J., Dion, P. A., St-Onge, J., Hince, P. et al. A mutation that creates a pseudoexon in SOD1 causes familial ALS. Ann. Hum. Genet. 73, 652-657 (2009).

$11 \mathrm{Zu}$, J. S., Deng, H. X., Lo, T. P., Mitsumoto, H., Ahmed, M. S., Hung, W. Y. et al. Exon 5 encoded domain is not required for the toxic function of mutant SOD1 but essential for the dismutase activity: identification and characterization of two new SOD1 mutations associated with familial amyotrophic lateral sclerosis. Neurogenetics 1, 65-71 (1997).

12 Dejesus-Hernandez, M., Kocerha, J., Finch, N., Crook, R., Baker, M., Desaro, P. et al. De novo truncating FUS gene mutation as a cause of sporadic amyotrophic lateral sclerosis. Hum. Mutat. 31, 1377-1389 (2010).

13 Nlend, R., Meyer, K. \& Schumperli, D. Repair of pre-mRNA splicing: Prospects for a therapy for Spinal Muscular Atrophy. RNA Biol. 7, 430-440 (2010).

14 Pros, E., Fernandez-Rodriguez, J., Benito, L., Ravella, A., Capella, G., Blanco, I. et al. Modulation of aberrant NF1 pre-mRNA splicing by kinetin treatment. Eur. J. Hum. Genet. 18, 614-617 (2009).

15 O'Leary, D. A., Vargas, L., Sharif, O., Garcia, M. E., Sigal, Y. J., Chow, S. K. et al. HTS-compatible patient-derived cell-based assay to identify small molecule modulators of aberrant splicing in myotonic dystrophy type 1. Curr. Chem. Genomics. 4, 9-18 (2010). 